\title{
Modeling and Applications of Space-Time Correlation for MIMO Fading Signals
}

\author{
Po-Ying Chen, Student Member, IEEE, and Hsueh-Jyh Li, Senior Member, IEEE
}

\begin{abstract}
In this paper, the space-time correlation (STC) functions of multiple-input multiple-output (MIMO) fading signals are derived for both frequency-flat and frequency-selective channels in macrocell and microcell environments. To avoid large computation load in calculating the exact STC, we recommend the concept of effective scatterers and provide simplified expressions of STC functions. The STC functions are used to calculate the coefficient matrices that are necessary for generating MIMO channels, when the vector autoregressive method is used. The analytical results are verified by Monte Carlo simulations. Finally, we use the generated channels to investigate MIMO capacities under various channel parameters and to evaluate the bit-error-rate performance of downlink wideband code-division-multiple-access system with transmit and receive diversity.
\end{abstract}

Index Terms-Channel modeling, multiple-input multipleoutput (MIMO), space-time correlation (STC).

\section{INTRODUCTION}

A S THE DEMAND on wireless multimedia service increases, high-data-rate transmission over wireless channels becomes more indispensable. To achieve the target data rate, one can simply shorten the symbol duration. This, however, implies that a larger frequency spectrum, which is at a premium, is required. Furthermore, the transmitting signals may suffer from severe intersymbol interference in a multipathplentiful environment. A more promising way is to use multiple antennas at the transmitters and the receivers. Such a system is often referred to as multiple-input multiple-output (MIMO) system. It was shown that under certain conditions, the capacity is proportional to the minimum number of transmitting and receiving antennas in a MIMO system [1]. Due to its performance boost and low cost (compared with the high-priced frequency spectrum), the multiantenna technology and, thereby, MIMO channel characteristics are concerned by more and more researchers recently.

It has been investigated in [2] that to what extent the MIMO capacity can reach greatly depends on the spatial correlation between radio links. When the mobility of users is taken into account, the fading characteristics of time-varying channels

Manuscript received February 13, 2006; revised July 5, 2006. This work was supported by the National Science Council of the Republic of China under Grant NSC 94-2219-E-002-003. The review of this paper was coordinated by Dr. K. Dandekar.

P.-Y. Chen is with Sunplus mMobile Inc., Hsinchu 300, Taiwan, R.O.C. (e-mail: r0942068@ee.ntu.edu.tw).

H.-J. Li is with the Graduate Institute of Communication Engineering and Department of Electrical Engineering, National Taiwan University, Taipei 10617, Taiwan, R.O.C. (e-mail: hjli@ew.ee.ntu.edu.tw).

Color versions of one or more of the figures in this paper are available online at http://ieeexplore.ieee.org.

Digital Object Identifier 10.1109/TVT.2007.897208 also influence the system performance. As a result, spatial and temporal channel statistics are essential for evaluating the performance of multiple-antenna communication systems. Traditionally, the spatial and the temporal correlations are regarded as separated effects, i.e., the space-time correlation (STC) function can be expressed as the product of spatial and temporal correlation functions. Under such an assumption, a sequence of random vectors with specific STCs can be generated by spatially transforming the set of independent and temporally filtered Gaussian random sequences [3]. In fact, it was shown that, for the scatterers of a ring model, the auto and the cross correlation of vector channels should be jointly determined by the spatial and the temporal separations [4]. Abdi and Kaveh [5] generalized this result to MIMO channels with nonisotropic scattering around the mobile station (MS) and derived a closed-form expression of the STC function. In [6], the model with scatterers on a ring was considered at both the MS and the base station (BS). Also, the effect of STC on MIMO capacity was further investigated. The previous works, however, assumed that the channel is frequency flat, i.e., all multipath signals arrive at the receiver within a symbol duration or, more specifically, a time bin (the reciprocal of transmission bandwidth). Up to now, the STC for each time-bin signal in frequency-selective fading channels has not been discussed to the best of authors' knowledge.

We generalize the related research and derive the STC expressions for the MIMO fading signals in macrocell and microcell environments. For the macrocells, the scatterers are assumed to be located uniformly inside a circular ring around MS. This scattering model has been applied in [7] to derive the space-time-frequency correlation of MIMO fading signals. For the microcells, by analogy with that in macrocells, we distribute scatterers inside an elliptical ring enclosing the MS and the BS. These two models can be, respectively, reduced to the well-known geometry-based single-bounce circular model (GBSBCM) and elliptical model (GBSBEM) proposed by Liberti and Rappaport [8]. In both environments, the influence of transmission bandwidth is taken into account. This results in narrowband (NB) and wideband (WB) MIMO fading channels. In order to reduce the large computation load for acquiring the exact STC, we derive simplified STC expressions by introducing the concept of effective scatterers. Moreover, the derived STCs are used to generate MIMO fading channels with the vector autoregressive (VAR) method [9]. In wireless channels, the scatterers often occur in clusters [10]. The additional clusters may be resulted from tall buildings in urban environments or high hills in rural areas. For such a scenario, we propose the concept of "image points" or, for example, "virtual mobiles," 


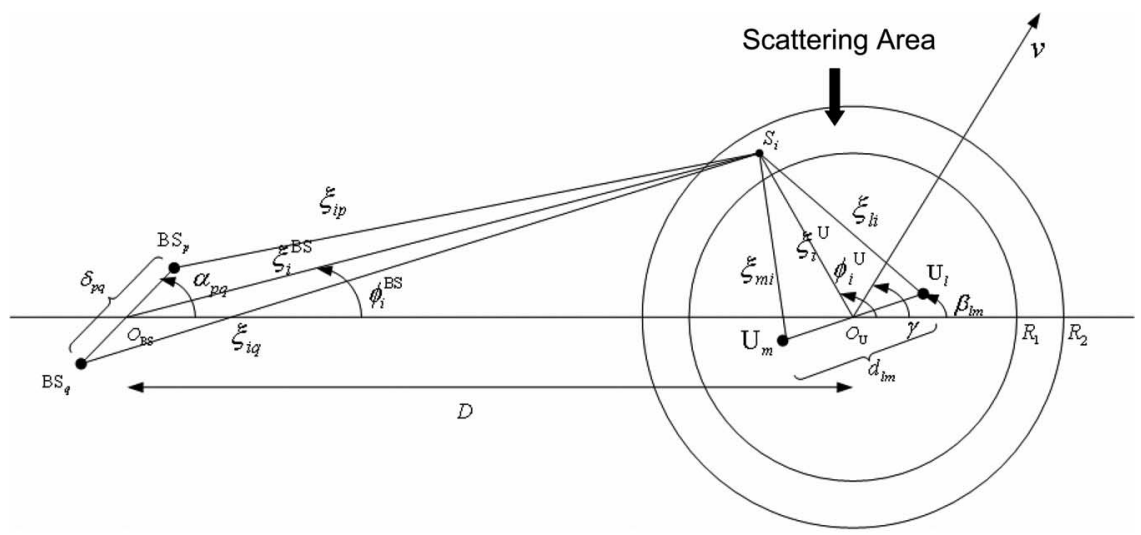

(a)

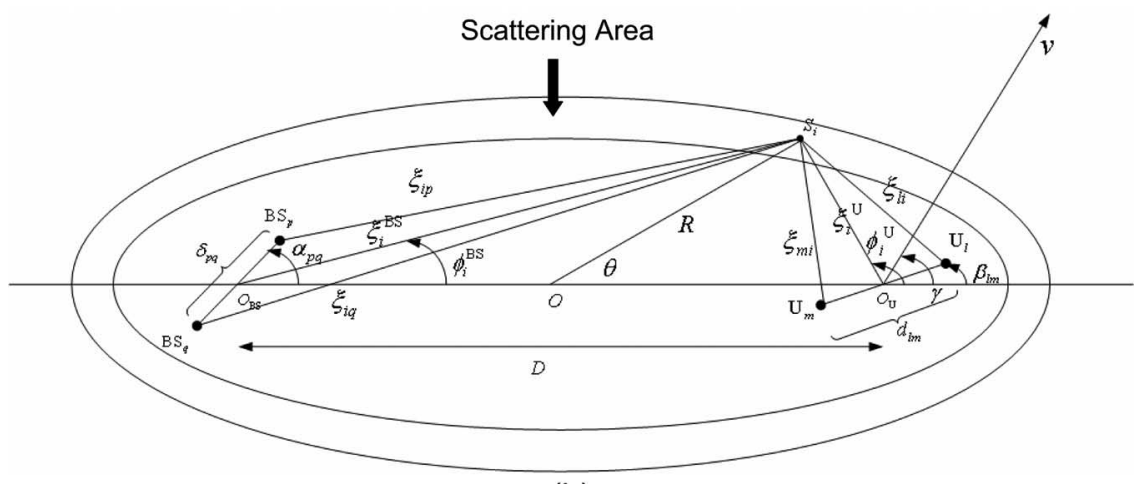

(b)

Fig. 1. Geometrical configuration of a $2 \times 2$ channel of the (a) circular ring (macrocells) and (b) elliptical ring (microcells) model.

which are induced by the additional clusters to synthesize the resulting fading channels. Throughout this paper, we focus on the spatial and temporal characteristics of the channels' scattering components. These results can be easily generalized to the scenario when a specular component exists.

The remainder of this paper is organized as follows. The signal model and the STC expressions for NB and WB fading signals in macrocell and microcell environments are presented in Section II. These results are used to generate MIMO fading signals using VAR method in Section III. In Section IV, we consider the scenario with multiple clusters in macrocells to retrieve the STC functions and generate MIMO fading channels. The analytical results are verified by Monte Carlo simulations in Section V, where the MIMO capacity and the bit-errorrate (BER) performance for downlink (DL) wideband codedivision-multiple-access (WCDMA) system are also evaluated by the generated time-varying channels. The conclusion is made in Section VI.

\section{STC FOR MIMO FADING SIGNALS}

In macrocell environments (GBSBCM), where the antenna height at BS is relatively high, scatterers are usually assumed to be uniformly distributed inside the circle around the MS. On the other hand, for microcells (GBSBEM), the multipath scattering is as likely near the BS as it is near the MS. Scatterers are assumed to be randomly distributed within an ellipse with BS and MS as its foci. This ellipse implicitly indicates the maximum delay spread among all multipath signals [8]. In the sequel, we assume that scatterers are uniformly distributed inside a circular or an elliptical ring to model the macrocells or microcells, respectively, as shown in Fig. 1. The circular ring model has been applied in [7] to represent macrocell environments. Besides, these two models are reduced to GBSBCM and GBSBEM when the area of inner circle or inner ellipse becomes very small.

We follow the same notations as those defined in [5], the baseband fading signal between the lth antenna element at MS and the $p$ th antenna element at BS can be represented by (1). The line-of-sight (LOS) component is neglected here for simplicity, although it can be easily generalized.

$$
\begin{aligned}
h_{l p}(t) & =\lim _{N \rightarrow \infty} \frac{1}{\sqrt{N}} \sum_{i=1}^{N} g_{i}\left(\xi_{i}^{\mathrm{BS}} \cdot \xi_{i}^{\mathrm{U}} / D\right)^{-n / 2} \\
\cdot & \exp \left\{j \varphi_{i}-j \frac{2 \pi}{\lambda}\left(\xi_{i p}+\xi_{l i}\right)+j 2 \pi f_{\mathrm{D}} t \cos \left(\phi_{i}^{\mathrm{U}}-\gamma\right)\right\}
\end{aligned}
$$

where $N$ is the number of independent scatterers $S_{i} . g_{i}$ and $\varphi_{i}$ represent the amplitude and phase shift introduced by the $i$ th scatterer, respectively. In (1), $\left\{g_{i}\right\}_{i=1}^{N}$ are independent positive random variables with finite variance; $\left\{\varphi_{i}\right\}_{i=1}^{N}$ are independently, identically, and uniformly distributed over $[0,2 \pi) . \xi_{\text {ip }}$ and $\xi_{l i}$ denote the distances shown in Fig. $1 . \phi_{i}^{\mathrm{U}}$ is the angle of arrival (AOA) of the wave traveling from the $i$ th scatterer 
toward the user. $f_{\mathrm{D}}$ is the maximum Doppler frequency, $\lambda$ is the wavelength, and the term $\left(\xi_{i}^{\mathrm{BS}} \cdot \xi_{i}^{\mathrm{U}} / D\right)^{-n}$ accounts for the power loss relative to the distance between the MS and the BS $D$ with path loss exponent $n$. The total power of this link is denoted as

$$
\Omega_{l p}=\lim _{N \rightarrow \infty} \frac{1}{N} \sum_{i=1}^{N} E\left\{g_{i}^{2}\right\}\left(\xi_{i}^{\mathrm{BS}} \cdot \xi_{i}^{\mathrm{U}} / D\right)^{-n} .
$$

Now, we define the normalized space-time crosscorrelational coefficient between the two pairs of channels $l$ to $p$ and $m$ to $q$ as

$$
\rho_{l p, m q}(\tau)=\frac{E\left\{h_{l p}(t) h_{m q}^{*}(t-\tau)\right\}}{\sqrt{\Omega_{l p} \Omega_{m q}}} .
$$

Let the scatterers be independently distributed according to some 2-D probability density function (PDF) $f_{\mathbf{S}}(\mathbf{s})$ on an area A. We plug (1) into (2) and assume equal power for all radio links, i.e., $\Omega_{l p}=\Omega_{m q}=\Omega$. Then, by the fact of $E\left\{g_{i}^{2}\right\} / N=f_{\mathbf{S}}(\mathbf{s}) d \mathbf{s}$, when $N$ becomes large, we can arrange the normalized STC as

$$
\begin{aligned}
\rho_{l p, m q}(\tau)= & \frac{1}{\Omega} \int_{\mathbf{A}}\left(\xi_{i}^{\mathrm{BS}} \cdot \xi_{i}^{\mathrm{U}} / D\right)^{-n} \\
& \cdot \exp \left\{-j 2 \pi / \lambda \cdot\left(\xi_{i p}-\xi_{i q}+\xi_{l i}-\xi_{m i}\right)\right. \\
& \left.\quad+j 2 \pi f_{\mathrm{D}} \tau \cos \left(\phi_{i}^{\mathrm{U}}-\gamma\right)\right\} f_{\mathbf{S}}(\mathbf{s}) d \mathbf{s} .
\end{aligned}
$$

In the following subsections, based on (3) and the corresponding geometries, we derive the STC expressions of NB fading signals and of each time-bin signal in a frequencyselective channel, under the environments of macrocell and microcell.

\section{A. STC for NB Fading Signals in Macrocells}

As indicated in Fig. 1(a), for the macrocell environment, the scatterers are assumed to be uniformly distributed inside the circular ring with inner and outer radius $R_{1}$ and $R_{2}$, respectively. We can reasonably assume $D \gg R_{2}>R_{1} \gg \max \left\{\delta_{p q}, d_{l m}\right\}$ and derive the normalized STC by integrating a modified Bessel function as (see Appendix I-A)

$$
\begin{aligned}
& \rho_{l p, m q}(\tau) \\
& \approx \frac{\exp \left\{j c_{p q} \cos \alpha_{p q}\right\}}{\Omega} \int_{R_{1}}^{R_{2}} \frac{2\left(\xi^{\mathrm{U}}\right)^{-n+1}}{R_{2}^{2}-R_{1}^{2}} \\
& \cdot I_{0}\left(\left\{-\left[a_{\tau} \sin \gamma+b_{l m} \sin \beta_{l m}+\left(\xi^{\mathrm{U}} / D\right) \cdot c_{p q} \sin \alpha_{p q}\right]^{2}\right.\right. \\
& \left.\left.\quad-\left[a_{\tau} \cos \gamma+b_{l m} \cos \beta_{l m}\right]^{2}\right\}^{\frac{1}{2}}\right) d \xi^{\mathrm{U}}
\end{aligned}
$$

where $a_{\tau}=2 \pi f_{\mathrm{D}} \tau, b_{l m}=2 \pi d_{l m} / \lambda, c_{p q}=2 \pi \delta_{p q} / \lambda$, and the zeroth order modified Bessel function is defined as

$$
\int_{-\pi}^{\pi} \exp (x \sin z+y \cos z) d z=2 \pi I_{0}\left(\sqrt{x^{2}+y^{2}}\right) .
$$

To calculate the STC of fading signals for GBSBCM, we force the radius of inner circle as a small positive value $\varepsilon$.

For simplicity, we use "effective" scatterers, which is the terminology first proposed in Lee's model [8], uniformly distributed around the ring with radius $R_{2}$ to represent the effect of many scatterers within the region. The resulting ring model is extensively used for simulation, channel characteristics study, or performance analysis. In the simplified model, we further neglect the path loss effect. Then, the integral in (4) can be taken out; this results in the compact closed form similar to the derived (12) in [5] with $K_{l p}=K_{m q}=0$ and $\kappa=0$ :

$$
\begin{aligned}
\rho_{i p, m q}(\tau) \approx & \frac{\exp \left\{j c_{p q} \cos \alpha_{p q}\right\}}{\Omega} \\
& \cdot I_{0}\left(\left\{-\left[a_{\tau} \sin \gamma+b_{l m} \sin \beta_{l m}+\Delta c_{p q} \sin \alpha_{p q}\right]^{2}\right.\right. \\
& \left.\left.-\left[a_{\tau} \cos \gamma+b_{l m} \cos \beta_{l m}\right]^{2}\right\}^{1 / 2}\right)
\end{aligned}
$$

where $\Delta$ is the angular spread at the BS defined as $\Delta=R_{2} / D$.

\section{B. STC for NB Fading Signals in Microcells}

In microcells, we assume that scatterers are distributed within the elliptical ring as shown in Fig. 1(b). The half lengths of the major axes of inner and outer ellipses are $a_{1}$ and $a_{2}$, which relate to the minimum and maximum delay time among all multipaths as $a_{1}=\left(D+c \cdot \tau_{\min }\right) / 2$ and $a_{2}=$ $\left(D+c \cdot \tau_{\max }\right) / 2$, respectively, where $c$ denotes the speed of light. The corresponding minor axis half lengths are $b_{i}=$ $\sqrt{a_{i}^{2}-D^{2} / 4}, i=1,2$. We use (3) with further arrangements (see Appendix I-B); the normalized STC can be obtained by

$$
\begin{gathered}
\rho_{l p, m q}(\tau) \\
\approx \frac{D^{n}}{\Omega} \int_{-\pi}^{\pi} \int_{r_{1}(\theta)}^{r_{2}(\theta)} \frac{R\left[\left(R^{2}+D^{2} / 4\right)^{2}-D^{2} R^{2} \cos ^{2} \theta\right]^{-n / 2}}{\pi\left(a_{2} b_{2}-a_{1} b_{1}\right)} \\
\cdot \exp \left\{\frac{j c_{p q}\left[R \cos \left(\alpha_{p q}-\theta\right)+D / 2 \cos \alpha_{p q}\right]}{\sqrt{R^{2}+D R \cos \theta+D^{2} / 4}}\right. \\
+\frac{j a_{\tau}[R \cos (\theta-\gamma)-D / 2 \cos \gamma]}{\sqrt{R^{2}-D R \cos \theta+D^{2} / 4}} \\
\left.+\frac{j b_{l m}\left[R \cos \left(\theta-\beta_{l m}\right)-D / 2 \cos \beta_{l m}\right]}{\sqrt{R^{2}-D R \cos \theta+D^{2} / 4}}\right\} d R d \theta
\end{gathered}
$$

where $r_{i}(\theta)=a_{i} b_{i} / \sqrt{b_{i}^{2} \cos ^{2} \theta+a_{i}^{2} \sin ^{2} \theta}, i=1,2$. 


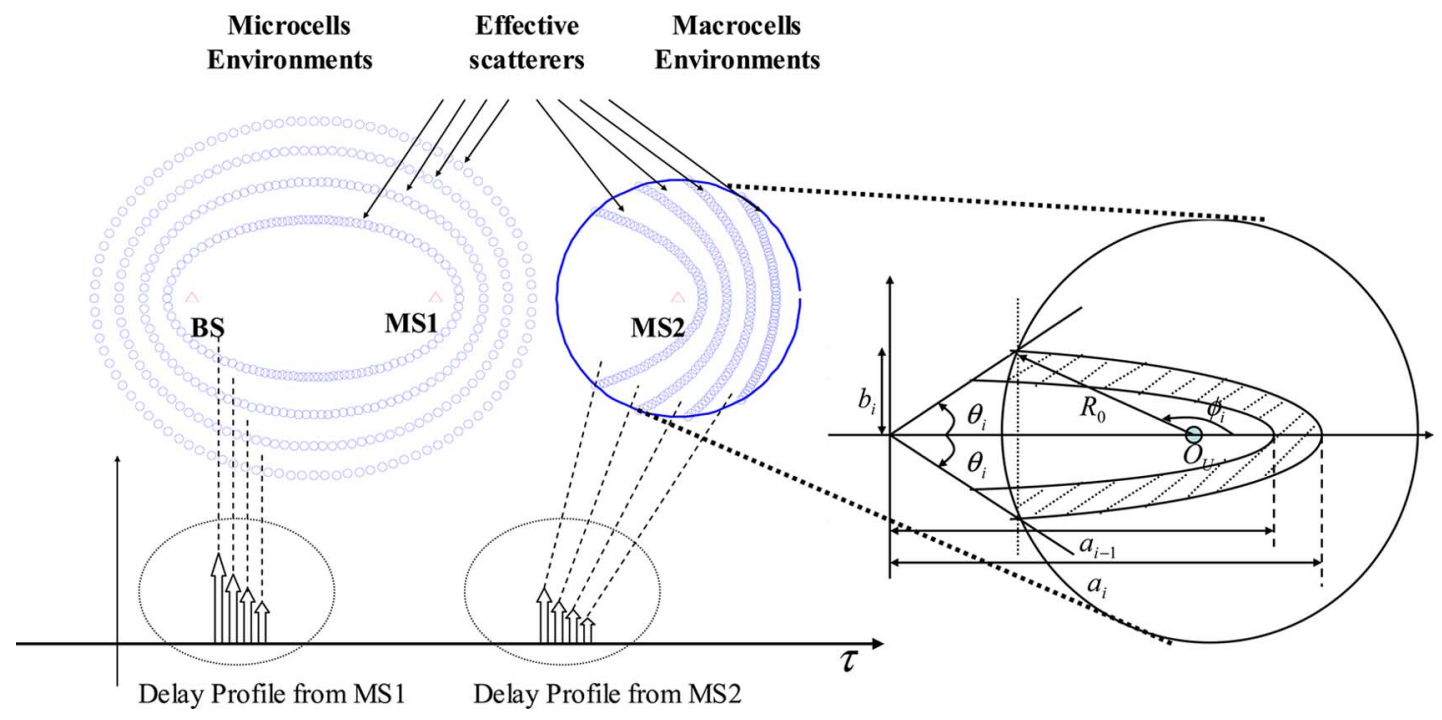

Fig. 2. Effective scatterers and the scattering region corresponding to each time-bin in frequency-selective channels.

To calculate the STC of fading signals for GBSBEM, we let the major axis half length of inner ellipse be a little greater than the half length of the distance between the BS and the MS, i.e., $a_{1}=D / 2+\varepsilon$, with $\varepsilon$ being a small positive value.

To reduce the complexity of calculating double integrals in (7), similar to the case in macrocells, we assume that the effective scatterers are uniformly distributed on the outer ellipse and make the path loss exponent $n$ equal to 0 . Then, the STC can be simplified by only calculating a single integral

$$
\begin{aligned}
\rho_{l p, m q}(\tau) \approx \frac{1}{2 \pi \Omega} \int_{-\pi}^{\pi} \exp \left\{\frac{j c_{p q}\left[\cos \left(\alpha_{p q}-\theta\right)+z \cos \alpha_{p q}\right]}{\sqrt{1+2 z \cos \theta+z^{2}}}\right. \\
+\frac{j b_{l m}\left[\cos \left(\theta-\beta_{l m}\right)-z \cos \beta_{l m}\right]}{\sqrt{1-2 z \cos \theta+z^{2}}} \\
\left.+\frac{j a_{\tau}[\cos (\theta-\gamma)-z \cos \gamma]}{\sqrt{1-2 z \cos \theta+z^{2}}}\right\} d \theta
\end{aligned}
$$

with $z=\left(D \sqrt{b_{2}^{2} \cos ^{2} \theta+a_{2}^{2} \sin ^{2} \theta}\right) / 2 a_{2} b_{2}$.

\section{STC for WB Fading Signals in Microcells}

When a wideband transmission is considered, numerous time-indifferentiable subpaths (TISs) will arrive at the receiver within a certain time-differentiable path (TDP) [3] or simply time-bin, which is the reciprocal of transmission bandwidth. Assuming that GBSBEM is applied in microcells, the scatterers that contribute to different TDPs will be located in different subregions, as illustrated by Fig. 2, where the effective scatterers are also plotted. Define $a_{0}=D / 2+\varepsilon$ and $b_{0}=\sqrt{a_{0}^{2}-D^{2} / 4}$, where $\varepsilon$ is a small positive value, then the scattering region that contribute to the $i$ th time-bin signal is enclosed by the two successive ellipses with major and minor axis half lengths, $a_{i-1}, b_{i-1}$, and $a_{i}, b_{i}$, respectively, where

$$
\left\{\begin{array}{l}
a_{i}=(D+i \cdot c \cdot \Delta \tau) / 2 \\
b_{i}=\sqrt{a_{i}^{2}-D^{2} / 4}
\end{array} \quad \text { for } i=1,2, \ldots, L\right.
$$

$L$ is the number of TDPs, and $\Delta \tau=1 / B$ with $B$ being the signal's transmission bandwidth. To acquire the STC function of the $i$ th time bin, we can calculate the integrals in (7) by replacing $a_{2}=a_{i}, b_{2}=b_{i}, a_{1}=a_{i-1}, b_{1}=b_{i-1}$ for the exact values or in (8) with $a_{2}=a_{i}$ and $b_{2}=b_{i}$ for simplified expression.

\section{STC for WB Fading Signals in Macrocells}

For the wideband transmission in GBSBCM, the scatterers that contribute to the $i$ th TDP are inside the intersection area of the ring with radius $R_{0}$ around the MS and the elliptical ring whose major and minor axis half lengths are $a_{i-1}, b_{i-1}$, and $a_{i}, b_{i}$ for the inner and the outer ellipse, respectively. $a_{i}$ and $b_{i}$ are the same as defined in (9). The scenario is also illustrated in Fig. 2.

To calculate the STC for the $i$ th time-bin signal, we directly use (7) and (8) with some modifications. The outer integral of (7) cannot be integrated over $[-\pi, \pi)$ here but over $\left[-\theta_{i}, \theta_{i}\right)$. As shown in the enlarged configuration in Fig. 2, $\theta_{i}$ can be calculated by (see Appendix II)

$$
\begin{aligned}
& \phi_{i}=\cos ^{-1}\left(\frac{b_{i}^{2} D-\sqrt{b_{i}^{4} D^{2}-\left(a_{i}^{2}-b_{i}^{2}\right)\left(4 a_{i}^{2} b_{i}^{2}-4 a_{i}^{2} R_{0}^{2}-b_{i}^{2} D\right)}}{2\left(a_{i}^{2}-b_{i}^{2}\right) R_{0}}\right) \\
& \theta_{i}=\tan ^{-1}\left(\frac{R_{0} \sin \phi_{i}}{D / 2+R_{0} \cos \phi_{i}}\right) .
\end{aligned}
$$

Similar to the previous cases, when the effective scatterers are assumed to be resided on the boundary of each subregion, the STC can be approximated by substituting $a, b$ with $a_{i}, b_{i}$ and $\pi$ with $\theta_{i}$ in (8).

\section{Generation of MiMO Fading Signals}

Since the derived STC function is not separable, the multipath vector channel simulator proposed in [3] is not feasible here. To generate the random variates with specific spatial and temporal correlations, the VAR method [9] is implemented. 
Consider the frequency-selective MIMO fading channel; let the $N_{r} \times N_{t}$ channel matrix corresponding to the lth TDP signal at time $t$ be

$\mathbf{H}(t, l)=\left[\mathbf{h}_{1}(t, l) \mathbf{h}_{2}(t, l) \cdots \mathbf{h}_{N_{t}}(t, l)\right], \quad$ for $l=1,2, \ldots, L$

where $\mathbf{h}_{i}(t, l)=\left[h_{1, i}(t, l) \quad h_{2, i}(t, l) \cdots h_{N_{r}, i}(t, l)\right]^{T}$ is the vector channel corresponding to the $i$ th transmitting antenna. The special case for $L=1$ corresponds to the frequencyflat fading channels. To generate the time-varying correlated MIMO channel for each time-bin $l$, we define the $N_{t} N_{r} \times 1$ vector as

$$
\mathbf{x}[n]=\operatorname{vec}\left(\mathbf{H}\left(n T_{s}, l\right)\right)=\left[\begin{array}{c}
\mathbf{h}_{1}\left(n T_{s}, l\right) \\
\mathbf{h}_{2}\left(n T_{s}, l\right) \\
\vdots \\
\mathbf{h}_{N_{t}}\left(n T_{s}, l\right)
\end{array}\right]
$$

and construct the correlation matrix whose entries are calculated by the simplified expression of STC function of each radio-link pair, as shown in (6) and (8), for ease of computation. The VAR parameter matrices are solved by the multichannel Yule-Walker equations [9].

Since we have omitted the path loss effect when deriving the simplified STC functions, all MIMO channels of different TDPs generated by this method have equal power, which is not the truth. Now, we redefine the power of each time-bin signal by taking into account the path loss as

$$
P_{l}=\frac{\int_{\mathbf{A}_{l}}\left(\xi_{i}^{\mathrm{BS}} \cdot \xi_{i}^{\mathrm{U}} / D\right)^{-n} f_{\mathbf{S}}(\mathbf{s}) d \mathbf{s}}{\sum_{k=1}^{L} \int_{\mathbf{A}_{k}}\left(\xi_{i}^{\mathrm{BS}} \cdot \xi_{i}^{\mathrm{U}} / D\right)^{-n} f_{\mathbf{S}}(\mathbf{s}) d \mathbf{s}}
$$

where $\mathbf{A}_{l}$ is the area occupied by the scatterers that contribute to the $l$ th TDP fading signal.

\section{Generalization to Multiple Clusters IN MACROCELLS}

In the environment of macrocells, some tall buildings or large obstacles may stand between the MS and the BS, thus result in additional clusters. In such a scenario, the transmitted signal from the MS may be reflected by the local scatterers around it, further by the construction, and then arrive at the BS. The portion of received radio wave reflected by a certain large obstacle can be viewed as that radiated from the point source which is located at the direction same as the reflector but with longer distance. Here, we call the virtual point source as the "image point" of the MS. The signal components that travel through twice reflections behave as if they were transmitted by the image point and reflected by the local scatterers around it, as illustrated in Fig. 3. Besides, when the mobile is moving, the image point also travels with the same speed but toward opposite direction. Since each image point behaves similar to the MS they emit, the same information source and travel at the same speed but with their own directions; the scatterers around

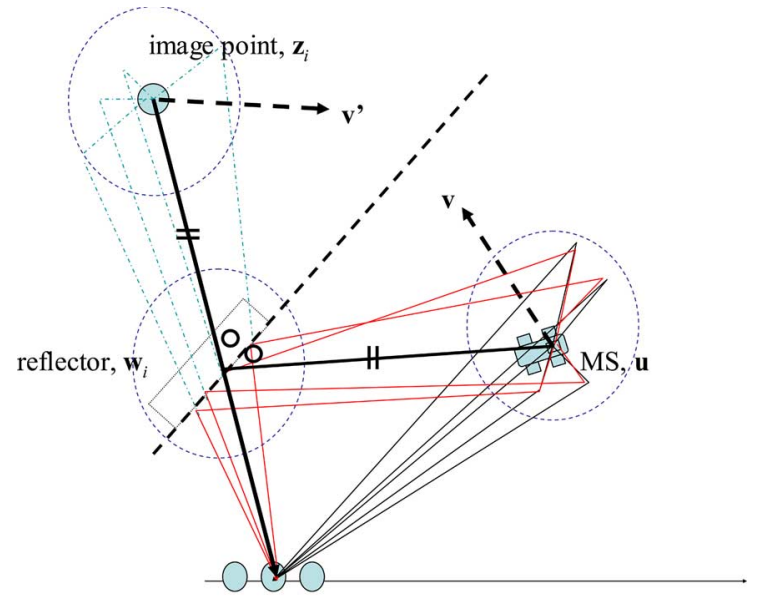

BS

Fig. 3. Scenario with two clusters in macrocells and the concept of image points.

the MS can be copied to the neighborhood of image points. We also name these image points as "virtual mobiles."

The vectors of positions and velocities of virtual mobiles can be computed by the geometry. On the $x-y$ plane spanned by the MS and the BS, we assume the BS is located at the origin, although it can be easily generalized for arbitrary locations. Let the position of the MS and the $i$ th large reflector be represented by vector $\mathbf{u}$ and $\mathbf{w}_{i}$ and the velocity of MS be $\mathbf{v}$ relative to the position of MS, as indicated in Fig. 3; then, the location of virtual MS $\mathbf{z}_{i}$ is represented by

$$
\mathbf{z}_{i}=\mathbf{w}_{i} \times\left(1+\frac{\left|\mathbf{u}-\mathbf{w}_{i}\right|}{\left|\mathbf{w}_{i}\right|}\right) .
$$

Let $\mathbf{q}=\left(\mathbf{z}_{i}+\mathbf{u}\right) / 2, \mathbf{x}=\left(\mathbf{q}-\mathbf{w}_{i}\right) /\left|\left(\mathbf{q}-\mathbf{w}_{i}\right)\right|$ and $\mathbf{y}$ be the vector orthogonal to $\mathbf{x}$, i.e., $\langle\mathbf{y}, \mathbf{x}\rangle=0$, then the velocity of the virtual MS can be calculated as

$$
\mathbf{v}_{i}^{\prime}=\langle\mathbf{v}, \mathbf{x}\rangle \cdot \mathbf{x}-\langle\mathbf{v}, \mathbf{y}\rangle \cdot \mathbf{y} .
$$

To compute the position of the $m$ th antenna element equipped at the virtual MS, we can simply replace $\mathbf{u}$ by $\mathbf{u}_{m}$, which denotes the location of the $m$ th antenna element on the mobile, in (14).

Based on the previous discussion, the location and velocity of each virtual MS can be obtained when the location information about the MS and the reflectors are given. For each (virtual) MS-BS pair, we can calculate the STCs by our derived formulas according to the geometry. Hence, the resulting normalized spatial-temporal correlation coefficient is the linear combination of the STCs contributed by each (virtual) MS-BS pair, i.e.,

$$
\rho_{l p, m q}(\tau)=\sum_{j=1}^{C} A_{j} \rho_{l p, m q}^{j}(\tau)
$$

where $C$ is the number of clusters (including the one around the MS), $\rho_{l p, m q}^{j}(\tau)$ is the normalized STC calculated from the geometry of the $j$ th (virtual) MS-BS pair, and the coefficient $A_{j}$ is related to the power contributed by the $j$ th cluster. Usually, $A_{j} \propto D_{j}^{-n}$ with $D_{j}$ denoting the distance from the virtual MS to the BS, and $\sum_{j=1}^{C} A_{j}=1$. 
By introducing the concept of virtual MS mentioned previously, one can use the VAR method described in the previous section to generate MIMO fading signals under the scenario with multiple clusters in the macrocell, which is a more realistic environment.

\section{VerificAtion, Applications, AND NUMERICAL RESULTS}

In this section, we first verify the derived theoretical STCs by comparing them with the correlation coefficients measured from Monte Carlo simulations. Then, two applications of utilizing the generated time-varying channels are presented: The MIMO capacity is investigated under different channel parameters, and finally, we evaluate the BER performance of DL-WCDMA system with transmit and receive diversity.

\section{A. STCs of MIMO Channels}

To evaluate the validity of the derived STC functions, we compare the analytical results with the correlation coefficients obtained from the 2-I-2-O fading channels that are generated by geometry- and VAR-based method. For the geometry-based channel simulator, we specify the locations of MS, BS, and scatterers, as indicated by the geometry shown in Fig. 1 and simply generate channel responses using (1) with $\{p, q\}=$ $\{l, m\}=\{2,1\}$. The center frequency is set as $2 \mathrm{GHz}$, which corresponds to the wavelength of $15 \mathrm{~cm}$. The spacing between antenna elements is $0.5 \lambda$. The initial locations of the MS, $\mathrm{X}$, for macrocells and microcells are $(300,1000)$ and $(100,400)$ on the $x-y$ plane, respectively. Besides, we let the BS be located at the origin, and its antenna elements lie along the $x$-axis; then, the angle between the BS array inline and the MS-BS line is $\alpha_{p q}=\pi-\angle \mathbf{X}$. The angle between the MS array inline and the MS-BS line $\beta_{l m}$ is set as $\pi / 4$ or $\pi / 8$ in macrocells or microcells. For both environments, the mobile is moving with a speed of $60 \mathrm{~km} / \mathrm{h}$, and we assume the MS is always moving along the MS array broadside direction, i.e., $\gamma=\beta_{l m}+\pi / 2$. The generated MIMO channels are sampled under sampling rate $f_{s}=1666.67 \mathrm{~Hz}$. The transmission bandwidth is $5 \mathrm{MHz}$, and the path loss exponent is 2. For GBSBEM, the maximum delay spread is set as $1 \mu \mathrm{s}$, and the radius of clusters $R_{0}$ for GBSBCM is $100 \mathrm{~m}$. We distribute 1000 scatterers every $10000 \pi \mathrm{m}^{2}$ approximately for both macrocells and microcells. For the VAR method, the AR matrices are calculated from the simplified STC expressions, and the regression order is chosen as 40.

The real part of the cross correlations of $h_{1,1}(t)$ and $h_{2,2}(t)$ is plotted in Fig. 4(a) and (b) under frequency-flat channels. In Fig. 4(c) and (d), the results are calculated from the second TDP of the WB fading signals in microcells and macrocells, respectively. From the simulation results, we can conclude that the simplified representations are close to the exact STCs except for the NB signal in microcells. This infers that the simplified STC for the first TDP of WB signals in microcells also deviates from the exact STC. This deviation between exact and simplified STC expressions is mainly due to the fact that GBSBEM and the simplified elliptical ring constructed by effective scatterers have different angular distributions seen by the MS or BS. This discrepancy, however, is not severe in macrocells according to the analytical results (not shown here). As we expect, these deviations will diminish as the transmission bandwidth increases. To sum up, for most cases, such as frequency-flat channels in macrocells and most time bins in the frequency-selective fading channels, the effective scatterers are representative of the averaged scattering environment; and the STC coefficients can be predicted by the simplified expressions.

\section{B. MIMO Capacity}

Let $\mathbf{H}_{t}$ denote a particular realization of the MIMO channel for NB transmission and $\mathbf{H}_{t, i}$ be the MIMO channel matrix of the $i$ th subchannel when the frequency band is divided into $N_{f}$ NB subchannels for WB transmission. It has been derived that the capacity of the MIMO channel in the absence of channel knowledge at the transmitter is given by $[11$, p. 83]

$$
C_{t}\left\{\begin{array}{l}
=\log _{2} \operatorname{det}\left(\mathbf{I}_{N_{r}}+\frac{\rho}{N_{t}} \mathbf{H}_{t} \mathbf{H}_{t}^{H}\right) \\
\approx \frac{1}{N_{f}} \sum_{i=1}^{N_{f}} \log _{2} \operatorname{det}\left(\mathbf{I}_{N_{r}}+\frac{\rho}{N_{t}} \mathbf{H}_{t, i} \mathbf{H}_{t, i}^{H}\right)
\end{array}\right.
$$

for NB and WB transmissions, respectively, where $\rho$ is the signal-to-noise ratio (SNR). To obtain the channel matrix of each subchannel for WB transmission, we generate the timevarying channel for each TDP and transform the impulse response to frequency response by $N_{f}$-points fast Fourier transform for each time instance.

In Fig. 5, the cumulative density functions (CDF) of the time-varying 2-I-2-O channel sequences for macrocells and microcells are plotted. The geometry and channel parameters for each environment are the same as those shown in the previous subsection, except that the bandwidth is set as $40 \mathrm{MHz}$ and $N_{f}=64$ for WB transmission to manifest the contribution of the frequency diversity. The results reveal that the MIMO channels generated by the two different methods-geometry- and VAR-based methods-have the same statistical characteristics (except the case of frequency-flat channel in microcells), thus implicitly verify our derived STC expressions. In the sequel, we use the VAR approach to generate the MIMO channels under different channel parameters and compare the outage capacities in frequency-flat and frequency-selective fading channels. The outage capacity $C_{\text {out }, p}$ is defined as the following: with probability $p$, the capacity of MIMO fading channels will fall below $C_{\text {out }, p}$, i.e., $P\left\{C_{t}<C_{\text {out }, p}\right\}=p$. We let $p=0.1$ in the following simulations.

In Fig. 6, we investigate the outage capacities under different antenna spacing at the MS $\left(d_{l m}\right)$ and the BS $\left(d_{p q}\right)$. As we expect, the increase of antenna spacing results in lower spatial correlation and, hence, higher capacity. In macrocells, the increase of $d_{p q}$ provides lower spatial correlation between antenna elements at the BS; therefore, the outage capacity keeps increasing with $d_{p q}$. However, it does not benefit from 
(a)

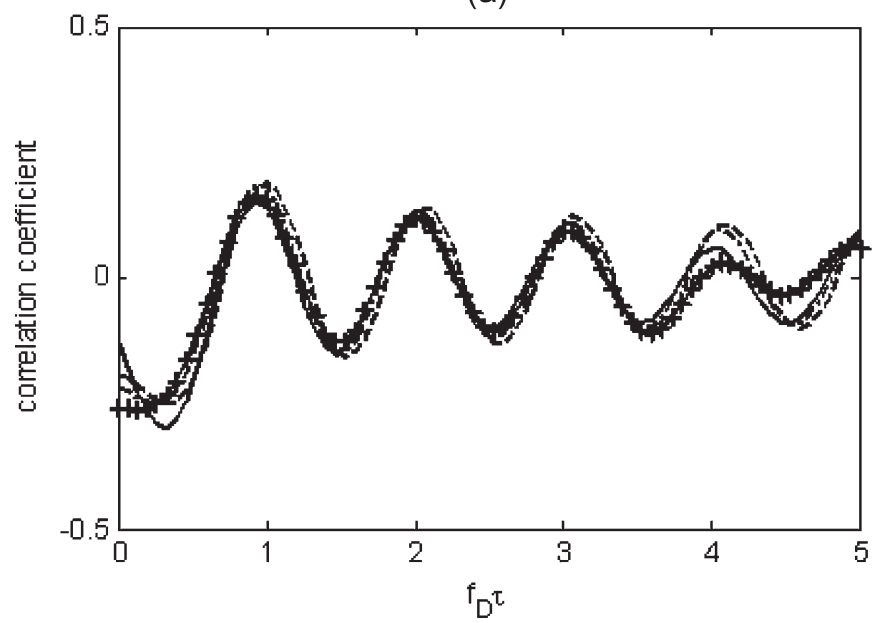

(b)

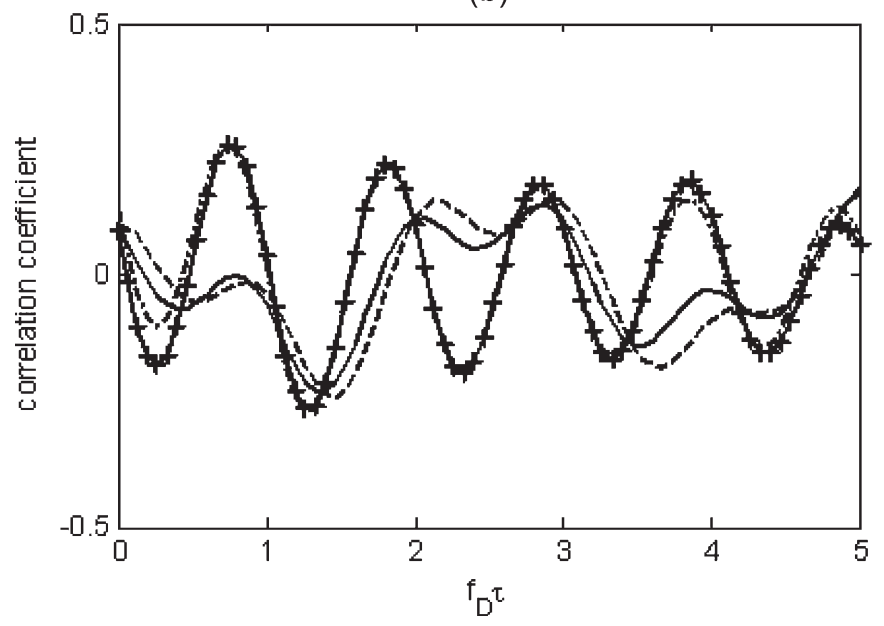

(c)

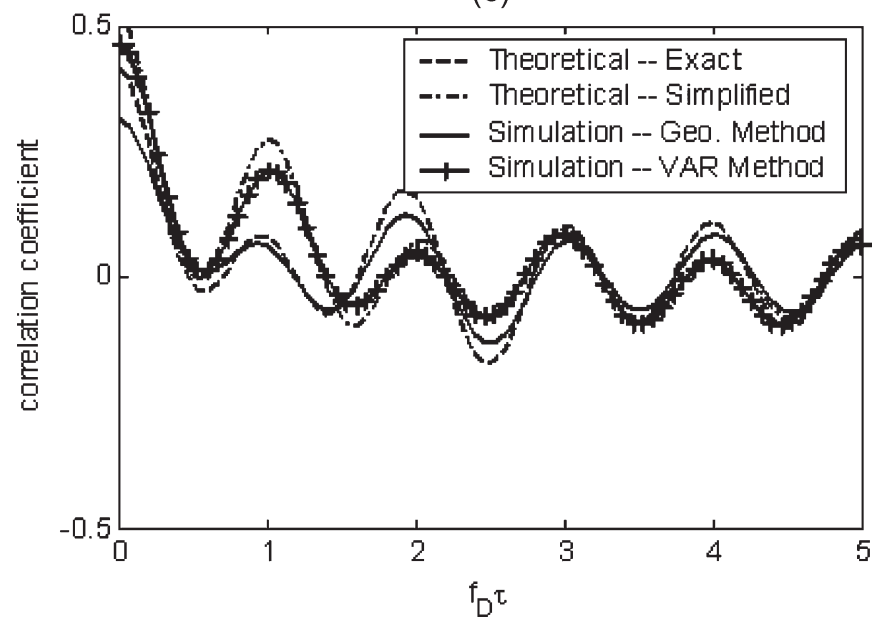

(d)

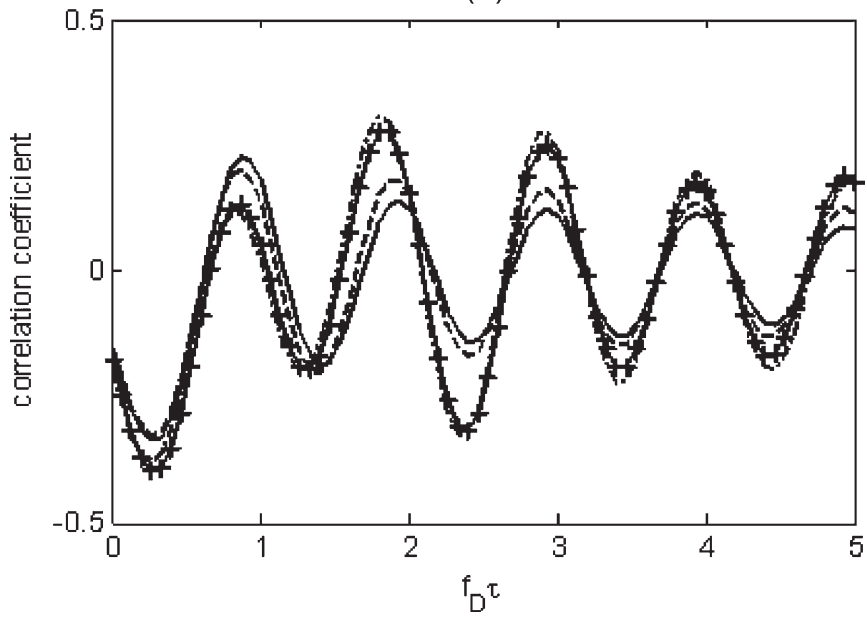

Fig. 4. Real part of the cross correlations of $h_{1,1}(t)$ and $h_{2,2}(t)$ of NB channels in (a) macrocells and (b) microcells and the second time bin of WB channels in (c) microcells and (d) macrocells.

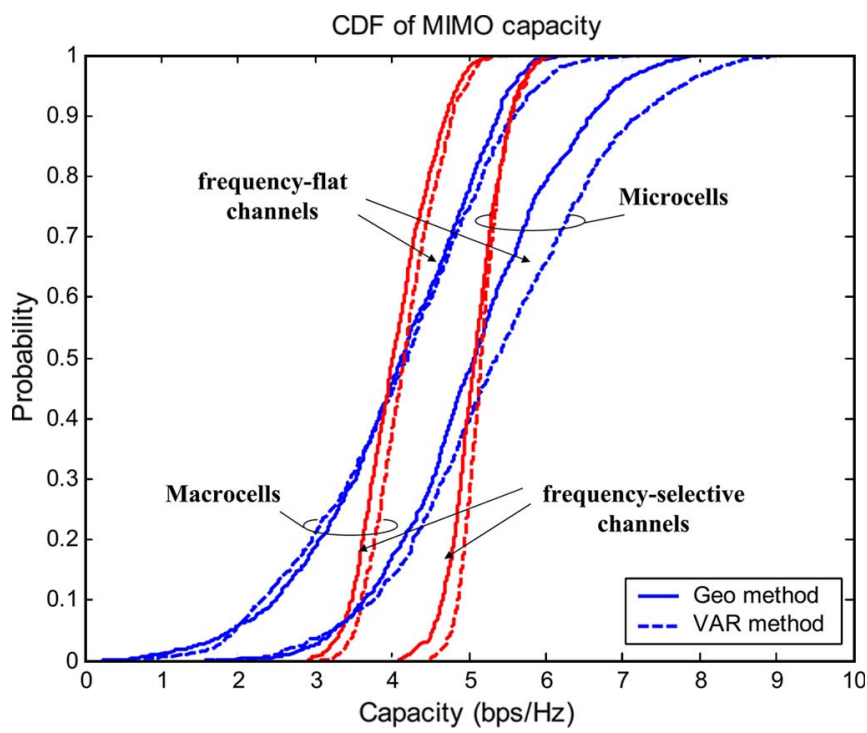

Fig. 5. CDFs of the 2-I-2-O channel capacities in (left) macrocells and (right) microcells.

increasing $d_{l m}$ after $d_{l m}>0.5 \lambda$. This is because the angular spread at the BS is much smaller than at the MS, where the multipaths uniformly come from all directions. For the microcell environments, since the scattering occurs in the neighborhood of the BS as well as the MS, small antenna spacing $(>0.5 \lambda)$ is enough to yield low spatial correlation and, hence, high capacity. In addition, the frequency diversity provides about $1 \mathrm{~b} / \mathrm{s} / \mathrm{Hz}$ capacity gain.

The impact of $\alpha_{p q}$ and $\beta_{l m}$ on the channel capacity is also studied. We vary the position of the mobile in the first quadrant on the $x-y$ plane with fixed MS-BS distance $D=1000 \mathrm{~m}$, thus results in different $\alpha_{p q} . \beta_{l m}$ is also changed from $0^{\circ}$ to $90^{\circ}$. Besides, the antenna spacing at both the MS and BS is $d_{l m}=\delta_{p q}=3 \lambda$ to stress the difference between simulation results. Other channel parameters remain the same as those described previously. Fig. 7 shows the MIMO capacity versus $\beta_{l m}$ when the mobile is located at different locations in macrocell environments. The simulation result indicates that the MIMO channel provides higher capacity when the MS moves closer to the broadside direction of the BS array, which agrees with the conclusion made in [2]. On the other hand, since the multipaths seen by the MS come from $[-\pi, \pi)$, the spatial correlation between the antenna elements remains low no matter how $\beta_{l m}$ changes. Therefore, the variation of $\beta_{l m}$ does not alter the capacity performance, as indicated by each horizontal line in this figure. 


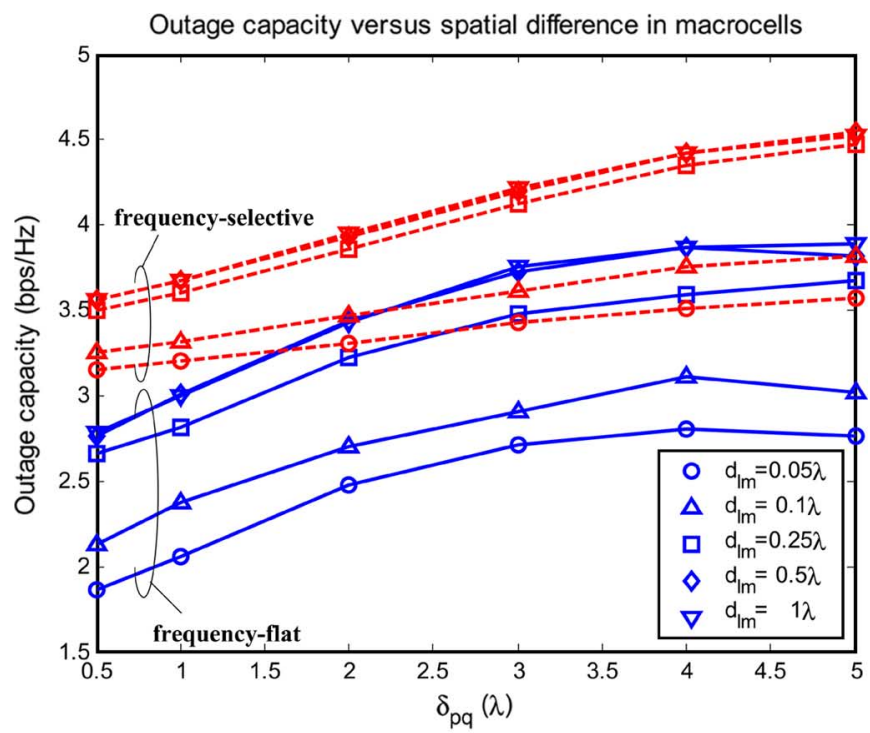

(a)

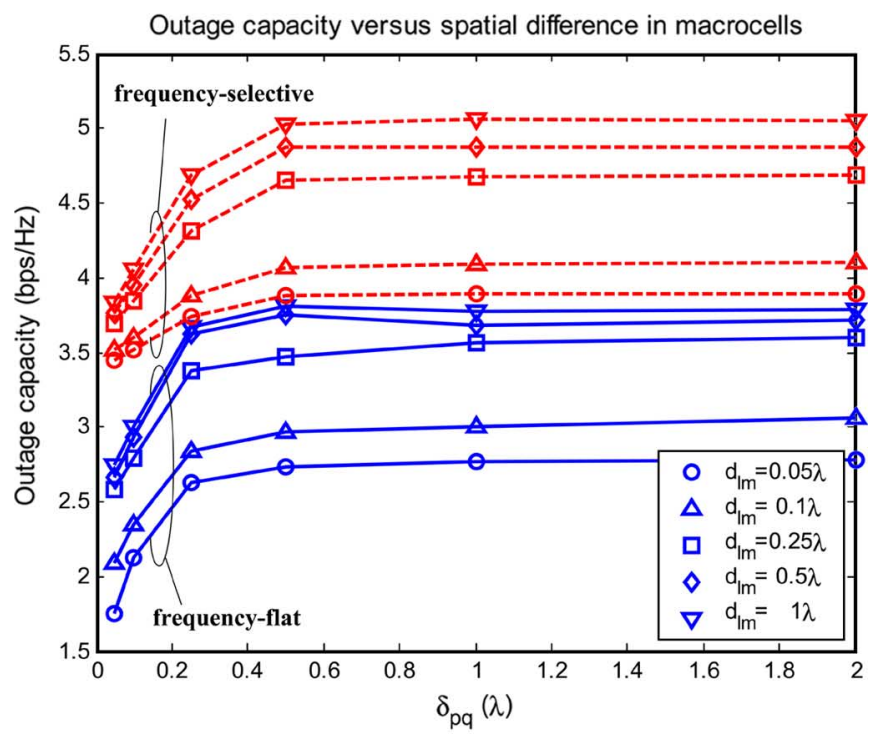

(b)

Fig. 6. Outage capacities versus spatial differences for frequency-flat or frequency-selective channels in (a) macrocells and (b) microcells.

\section{Performance of DL-WCDMA With Tx. and Tx. Diversity}

Before leaving this section, we evaluate the performance of DL-WCDMA system with two antennas at both the MS and the BS. There are two approaches, which are specified in the 3GPP standards [12], [13], to implement the transmit diversity-open and closed loops. In the open loop scheme, no channel state information (CSI) is known to the transmitter. It uses a simple space-time block code proposed by Alamouti [14] at the BS. As for the closed loop approach, the same information is transmitted through both antennas after multiplying individual antenna weights calculated according to the feedback CSI. Although the receive diversity is not defined in the standards, here, we use maximal ratio combining (MRC) at the mobile terminal, which is the same scheme as used in [15] for analyzing the BER performance.

In our simulation, we assume that the perfect CSI without quantization is available to both the receiver and the transmitter

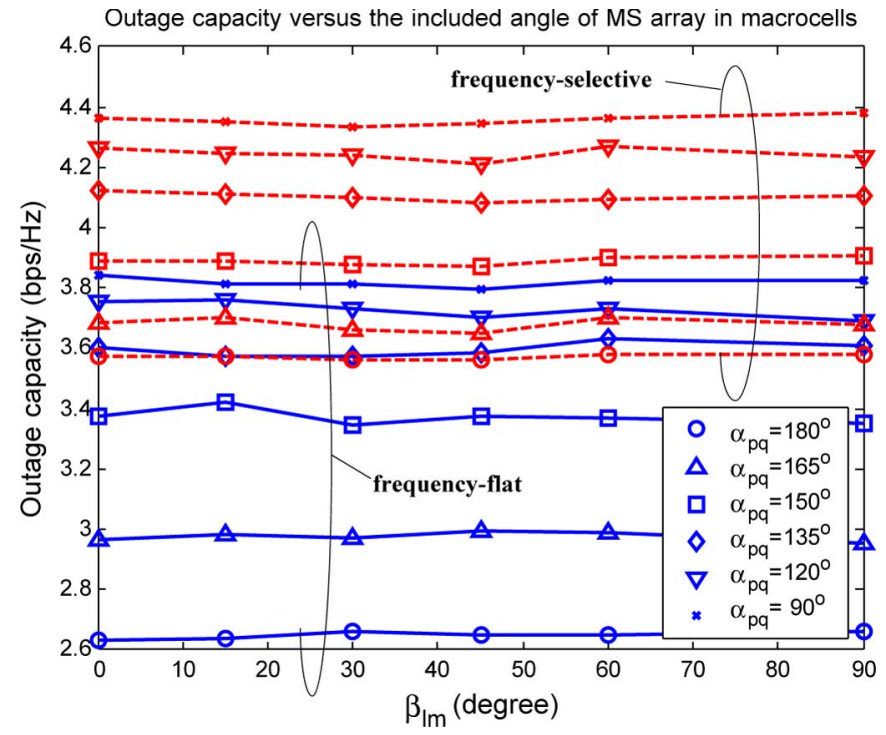

Fig. 7. Outage capacities versus $\beta_{l m}$ for frequency-flat or frequency-selective channels in macrocells.

in the closed loop scheme. The normalized antenna weight vector is selected as the eigenvector corresponding to the largest eigenvalue of the matrix $\mathbf{H}^{H} \mathbf{H}$. $\mathbf{H}$ is the channel matrix obtained by concatenating channel impulse responses of all MIMO radio links, given by [15]

$$
\mathbf{H}=\left[\begin{array}{ll}
\mathbf{h}_{11} & \mathbf{h}_{21} \\
\mathbf{h}_{12} & \mathbf{h}_{22}
\end{array}\right]
$$

where $\mathbf{h}_{i j}=\left[\begin{array}{llll}h_{i j, 1} & h_{i j, 2} & \cdots & h_{i j, L}\end{array}\right]^{T}$ is the impulse response between the $i$ th transmitting and the $j$ th receiving antenna. At the receiver, the RAKE receiver with $L$ fingers is implemented after the MRC.

Fig. 8 shows BER performance of the DL-WCDMA with transmit and receive diversity under three different channel scenarios: macrocells with single cluster, microcells, and macrocells with two clusters from left to right successively. The additional cluster in the third scenario results from a large reflector located at $(-300,400)$ on the $x-y$ plane. Other channel parameters are specified before. In each figure, the singlepath independently and identically distributed (i.i.d.) Rayleigh fading channel without spatial correlation is also evaluated for comparison. The simulation results show that the closed loop provides $3-\mathrm{dB}$ improvement over the open loop scheme, which agrees with the analytical results in [15]. In macrocells, since there exits high correlation between two antenna elements at the BS, the performance is worse than the i.i.d. Rayleigh channel case. Here, the RAKE receiver does not contribute much path diversity because the energy concentrates mostly on the first TDP. For the microcell environments, not only the correlation between antennas is low enough to provide almost the same spatial diversity as the i.i.d. channels, but the additional path diversity improves the performance. When the additional cluster is added in the macrocells, the angular spread at the BS increases as well as the delay spread. Hence, the 


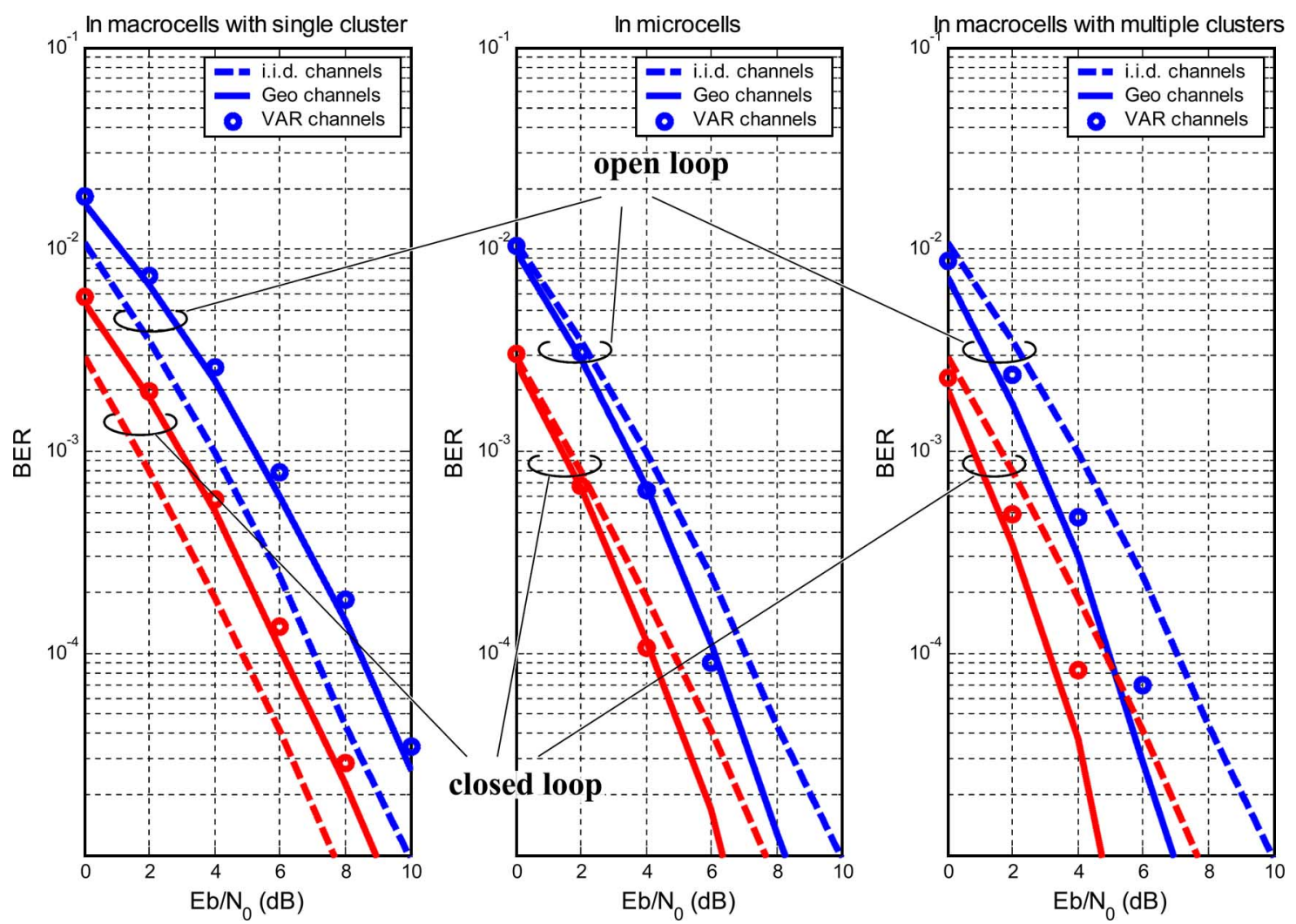

Fig. 8. BER performance of the DL-WCDMA with transmit and receive diversity in macrocells with single cluster in microcells and in macrocells with two clusters (from left to right).

system can benefit from both the spatial and path diversity concurrently. Finally, we find that the BER curves simulated over the channels generated by the two different approaches, geometry- and VAR-based method, are quite matched; this again shows the validity of our derived STC functions and the proposed concepts.

\section{CONCLUSION}

In this paper, the STC functions for MIMO fading channels in macrocells and microcells are derived. In both scenarios, NB and wideband transmissions are considered. We apply the derived STC functions to calculate the AR coefficient matrices for generating MIMO time-varying channels using the VAR method. The concept of "virtual mobiles" facilitates the generalization to the scenario with multiple clusters in macrocells. In addition to the verification of STC functions by Monte Carlo simulation results, we also investigate the MIMO capacity in different environments and MS-BS geometries. The derived STC functions are not only useful in analyzing the channel characteristics but can be applied to generate MIMO timevarying channels efficiently for simulation purpose.

\section{APPENDIX I}

In this appendix, we derive the STC function of NB MIMO fading signals in macrocells and microcells. The cases of frequency-selective fading channel can be easily generalized from these results with some modifications or variable substitutions.

\section{A. Derivation of the STC for NB Fading Signals in Macrocells}

As the configuration shown in Fig. 1(a), where we assume the scatterers are randomly distributed inside the area of circular ring around the MS, then the STC in (3) can be written as

$$
\begin{aligned}
& \rho_{l p, m q}(\tau)=\frac{1}{\Omega} \int_{R_{1}}^{R_{2}} \int_{-\pi}^{\pi}\left(\xi_{i}^{\mathrm{BS}} \cdot \xi^{\mathrm{U}} / D\right)^{-n} \\
& \cdot \exp \left\{-j \frac{2 \pi}{\lambda}\left(\xi_{i p}-\xi_{i q}+\xi_{l i}-\xi_{m i}\right)+j 2 \pi f_{\mathrm{D}} \tau \cos \left(\phi^{\mathrm{U}}-\gamma\right)\right\} \\
& \times f\left(\phi^{\mathrm{U}}, \xi^{\mathrm{U}}\right) d \phi^{\mathrm{U}} d \xi^{\mathrm{U}}
\end{aligned}
$$

where the notation of each variable is indicated in the figure. $f\left(\phi^{\mathrm{U}}, \xi^{\mathrm{U}}\right)$ is the PDF of the locations of scatterers relative to the MS with AOA $\phi^{\mathrm{U}}$ and distance $\xi^{\mathrm{U}}$. $\xi_{i}^{\mathrm{BS}}$, and $\xi_{i p}$, both determined by $\phi^{\mathrm{U}}$ and $\xi^{\mathrm{U}}$, are the distances from a certain scatterer $S_{i}$ inside the circular ring to the location of BS and the $p$ th antenna elements at the BS, respectively; and so on. According to law of cosines and sines, we can write the relations as

$$
\begin{gathered}
\xi_{i p}^{2}=\frac{\delta_{p q}^{2}}{4}+\xi_{i}^{\mathrm{BS}^{2}}-\delta_{p q} \xi_{i}^{\mathrm{BS}} \cos \left(\alpha_{p q}-\phi_{i}^{\mathrm{BS}}\right) \\
\xi_{i q}^{2}=\frac{\delta_{p q}^{2}}{4}+\xi_{i}^{\mathrm{BS}^{2}}+\delta_{p q} \xi_{i}^{\mathrm{BS}} \cos \left(\alpha_{p q}-\phi_{i}^{\mathrm{BS}}\right) \\
\xi_{l i}^{2}=\frac{d_{l m}^{2}}{4}+\xi^{\mathrm{U}^{2}}-d_{l m} \xi^{\mathrm{U}} \cos \left(\phi^{\mathrm{U}}-\beta_{l m}\right) \\
\xi_{m i}^{2}=\frac{d_{l m}^{2}}{4}+\xi^{\mathrm{U}^{2}}+d_{l m} \xi^{\mathrm{U}} \cos \left(\phi^{\mathrm{U}}-\beta_{l m}\right)
\end{gathered}
$$


and

$$
\frac{D}{\sin \left(\phi^{\mathrm{U}}-\phi_{i}^{\mathrm{BS}}\right)}=\frac{\xi^{\mathrm{U}}}{\sin \phi_{i}^{\mathrm{BS}}}=\frac{\xi_{i}^{\mathrm{BS}}}{\sin \phi^{\mathrm{U}}}
$$

Since the appropriate assumption of $D \gg R_{2}>R_{1} \gg$ $\max \left\{\delta_{p q}, d l m\right\}$, the differences of path lengths contributed by a certain scatterer can be approximated as

$$
\begin{aligned}
& -\left(\xi_{i p}-\xi_{i q}\right) \approx \delta_{p q} \cos \left(\alpha_{p q}-\phi_{i}^{\mathrm{BS}}\right) \\
& -\left(\xi_{l i}-\xi_{m i}\right) \approx d_{l m} \cos \left(\phi^{\mathrm{U}}-\beta_{l m}\right)
\end{aligned}
$$

since $\left(\xi_{i p}+\xi_{i q}\right) \approx 2 \xi_{i}^{\mathrm{BS}}$ and $\left(\xi_{l i}+\xi_{m i}\right) \approx 2 \xi^{\mathrm{U}}$. The first equation in (22) can be further approximated as

$$
-\left(\xi_{i p}-\xi_{i q}\right) \approx \delta_{p q}\left(\cos \alpha_{p q}+\frac{\xi^{\mathrm{U}}}{D} \sin \alpha_{p q} \sin \phi^{\mathrm{U}}\right)
$$

Substituting the arguments in (19) with the previous equations yields

$$
\begin{aligned}
& \rho_{l p, m q}(\tau) \approx \frac{1}{\Omega} \int_{R_{1}}^{R_{2}} \int_{-\pi}^{\pi} \xi^{\mathrm{U}^{-n}} \\
& \cdot \exp \left\{j \frac{2 \pi}{\lambda} \delta_{p q}\left[\cos \alpha_{p q}+\frac{\xi^{\mathrm{U}}}{D} \sin \alpha_{p q} \sin \phi^{\mathrm{U}}\right]\right. \\
&+j \frac{2 \pi}{\lambda} d_{l m} \cos \left(\phi^{\mathrm{U}}-\beta_{l m}\right) \\
&\left.\quad+j 2 \pi f_{\mathrm{D}} \tau \cos \left(\phi^{\mathrm{U}}-\gamma\right)\right\} \\
& \times \frac{\xi^{\mathrm{U}}}{\pi\left(R_{2}^{2}-R_{1}^{2}\right)} d \phi^{\mathrm{U}} d \xi^{\mathrm{U}}
\end{aligned}
$$

where we use the PDF as $f\left(\phi^{\mathrm{U}}, \xi^{\mathrm{U}}\right)=\xi^{\mathrm{U}} / \pi\left(R_{2}^{2}-R_{1}^{2}\right)$, which indicates that the scatterers are uniformly distributed inside the circular ring. According to the modified Bessel function defined in (5), we rearrange the previous equation and derive the STC function shown in (4).

Once the effective scatterers are assumed to be located on the ring with radius $R_{2}$ and the path loss effect is neglected, the STC in (19) is rewritten as

$$
\begin{aligned}
\rho_{l p, m q}(\tau)=\frac{1}{\Omega} \int_{-\pi}^{\pi} & \exp \left\{-j(2 \pi / \lambda) \cdot\left(\xi_{i p}-\xi_{i q}+\xi_{l i}-\xi_{m i}\right)\right. \\
& \left.+j 2 \pi f_{\mathrm{D}} \tau \cos \left(\phi^{\mathrm{U}}-\gamma\right)\right\} f\left(\phi^{\mathrm{U}}\right) d \phi^{\mathrm{U}} .
\end{aligned}
$$

With uniformly distributed scatterers as $f\left(\phi^{\mathrm{U}}\right)=1 / 2 \pi$ and the approximations in (22) and (23), the resulting compact closed form of the STC function is represented in (6).

\section{B. Derivation of the STC for NB Fading Signals in Microcells}

For the case of microcells as shown in Fig. 1(b), we assume the antenna elements are closely spaced at both the MS and the BS, i.e., $\xi_{i}^{\mathrm{BS}} \gg \delta_{p q}, \xi_{i}^{\mathrm{U}} \gg d_{l m}$. This is somewhat reasonable when the carrier frequency is high since a small size of antenna array can be achieved. With such an assumption, the same approximations as (22) can be made. To facilitate the derivation, we use polar coordinate with respect to the center point between MS and BS and substitute the arguments with $R$ and $\theta$, as denoted in Fig. 1. Again, by means of the law of cosines for triangles $O_{\mathrm{BS}} S_{i} O$ and $O_{\mathrm{U}} S_{i} O$, we have

$$
\begin{aligned}
\xi_{i}^{\mathrm{BS}^{2}} & =R^{2}+D R \cos \theta+D^{2} / 4 \\
\xi_{i}^{\mathrm{U}^{2}} & =R^{2}-D R \cos \theta+D^{2} / 4 .
\end{aligned}
$$

Besides, the angles $\phi_{i}^{\mathrm{BS}}$ and $\phi_{i}^{\mathrm{U}}$ can be related to the arguments $R$ and $\theta$ by

$$
\left\{\begin{aligned}
\sin \phi_{i}^{\mathrm{BS}} & =R \sin \theta / \xi_{i}^{\mathrm{BS}} \\
& =R \sin \theta / \sqrt{R^{2}+D R \cos \theta+D^{2} / 4} \\
\cos \phi_{i}^{\mathrm{BS}} & =(R \cos \theta+D / 2) / \xi_{i}^{\mathrm{BS}} \\
& =(R \cos \theta+D / 2) / \sqrt{R^{2}+D R \cos \theta+D^{2} / 4}
\end{aligned}\right.
$$

and

$$
\left\{\begin{array}{rl}
\sin \phi_{i}^{\mathrm{U}} & =R \sin \theta / \xi_{i}^{\mathrm{U}} \\
& =R \sin \theta / \sqrt{R^{2}-D R \cos \theta+D^{2} / 4} \\
\cos \phi_{i}^{\mathrm{U}} & =(R \cos \theta-D / 2) / \xi_{i}^{\mathrm{U}} \\
& =(R \cos \theta-D / 2) / \sqrt{R^{2}-D R \cos \theta+D^{2} / 4}
\end{array} .\right.
$$

Using the trigonometric addition formula, $\cos (x-y)=$ $\cos x \cos y+\sin x \sin y$, and (22), with some further rearrangements, the components inside the exponential function are represented as

$$
\begin{array}{r}
-\left(\xi_{i p}-\xi_{i q}+\xi_{l i}-\xi_{m i}\right) \\
\approx \frac{\delta_{p q}\left[R \cos \left(\alpha_{p q}-\theta\right)+D \cos \alpha_{p q} / 2\right]}{\sqrt{R^{2}+D R \cos \theta+D^{2} / 4}} \\
+\frac{d_{l m}\left[R \cos \left(\theta-\beta_{l m}\right)-D \cos \beta_{l m} / 2\right]}{\sqrt{R^{2}-D R \cos \theta+D^{2} / 4}}
\end{array}
$$

and

$$
\cos \left(\phi^{\mathrm{U}}-\gamma\right)=\frac{R \cos (\theta-\gamma)-D \cos \gamma / 2}{\sqrt{R^{2}-D R \cos \theta+D^{2} / 4}}
$$

For uniformly scattering inside the elliptical ring enclosing the MS and the BS, the PDF is

$$
f(R, \theta)=\frac{R}{\pi\left(a_{2} b_{2}-a_{1} b_{1}\right)} .
$$

Finally, simply plugging (29)-(31) into (3) yields the STC formula as shown in (7), where the upper and lower 
limits - $r_{1}(\theta), r_{2}(\theta)$ - of inner integral indicate the boundaries of inner and outer ellipses corresponding to angle $\theta$, respectively.

While only the effective scatterers located on the outer ellipse are taken into account, the inner integral of (7) can be removed, and the PDF is replaced by $f(\theta)=1 / 2 \pi$. In addition, the arguments $R$ inside the integrand should be replaced by a function of $\theta$ as

$$
r(\theta)=\frac{a_{2} b_{2}}{\sqrt{b_{2}^{2} \cos ^{2} \theta+a_{2}^{2} \sin ^{2} \theta}} .
$$

By defining $z=D / 2 r(\theta)$, the STC expression after the simplified form shown in (8) can be easily derived.

\section{APPENDIX II}

In this appendix, we derive the spreading angle $\theta_{i}$ which serves as the upper limit when computing the STCs of the $i$ th time-bin signal in frequency-selective channels in macrocells. As shown in the enlarged configuration of Fig. 2, we first calculate the value of $\phi_{i}$, which is the angle of the intersection point relative to the position of the MS, by the relation of

$$
\frac{\left(R_{0} \cos \phi_{i}+D / 2\right)^{2}}{a_{i}^{2}}+\frac{R_{0}^{2} \sin ^{2} \phi_{i}}{b_{i}^{2}}=1 .
$$

The problem here is to solve the quadratic equation in variable of $\cos \phi_{i}$ as indicated by

$$
\begin{aligned}
\left(a_{i}^{2}-b_{i}^{2}\right) R_{0}^{2} \cos ^{2} \phi_{i}-b_{i}^{2} D R_{0} \cos \phi_{i} \\
\quad+\left(a_{i}^{2} b_{i}^{2}-a_{i}^{2} R_{0}^{2}-b_{i}^{2} D^{2} / 4\right)=0
\end{aligned}
$$

and the solution is

$$
\cos \phi_{i}=\frac{b_{i}^{2} D-\sqrt{b_{i}^{4} D^{2}-\left(a_{i}^{2}-b_{i}^{2}\right)\left(4 a_{i}^{2} b_{i}^{2}-4 a_{i}^{2} R_{0}^{2}-b_{i}^{2} D^{2}\right)}}{2 R_{0}\left(a_{i}^{2}-b_{i}^{2}\right)}
$$

where we have taken off the plus sign in the numerator to avoid meaningless case of $\cos \phi_{i}>1$. Furthermore, using the law of sines, $\theta_{i}$ can be related to $\phi_{i}$ as

$$
\frac{D / 2}{\sin \left(\phi_{i}-\theta_{i}\right)}=\frac{R_{0}}{\sin \theta_{i}} .
$$

We substitute $\sin \left(\phi_{i}-\theta_{i}\right)=\sin \phi_{i} \cos \theta_{i}-\cos \phi_{i} \sin \theta_{i}$ into (36) and organize it as

$$
\tan \theta_{i}=\frac{R_{0} \sin \phi_{i}}{D / 2+R_{0} \cos \phi_{i}}
$$

which results in the second equation of (10).

\section{REFERENCES}

[1] E. Telatar, "Capacity of multi-antenna Gaussian channels," Eur. Trans. Commun., vol. 10, no. 6, pp. 585-595, Nov./Dec. 1999.

[2] D. S. Shiu, G. J. Foschini, M. J. Gans, and J. M. Kahn, "Fading correlation and its effect on the capacity of multi-element antenna systems," IEEE Trans. Commun., vol. 48, no. 3, pp. 502-513, Mar. 2000.
[3] A. Stéphenne and B. Champagne, "Effective multi-path vector channel simulator for antenna array systems," IEEE Trans. Veh. Technol., vol. 49, no. 6, pp. 2370-2381, Nov. 2000

[4] T. A. Chen, M. P. Fitz, W. Y. Kuo, M. D. Zoltowski, and J. G. Grimm, "A space-time model for frequency nonselective Rayleigh fading channels with applications to space-time modems," IEEE J. Sel. Areas Commun., vol. 18, no. 7, pp. 1175-1190, Jul. 2000.

[5] A. Abdi and M. Kaveh, "A space-time correlation model for multielement antenna systems in mobile fading channels," IEEE J. Sel. Areas Commun., vol. 20, no. 3, pp. 550-560, Apr. 2002.

[6] G. J. Byers and F. Takawira, "Spatially and temporally correlated MIMO channels: Modeling and capacity analysis," IEEE Trans. Veh. Technol., vol. 53, no. 3, pp. 634-643, May 2004.

[7] Z. Latinovic, A. Abdi, and Y. Bar-Ness, "A wideband space-time model for MIMO mobile fading channels," in Proc. IEEE WCNC, 2003 , pp. 338-342.

[8] J. C. Liberti and T. S. Rappaport, Smart Antennas for Wireless Communications: IS-95 and Third Generation CDMA Applications. Englewood Cliffs, NJ: Prentice-Hall, 1999

[9] K. E. Baddour and N. C. Beaulieu, "Accurate simulation of multiple crosscorrelated Rician fading channels," IEEE Trans. Commun., vol. 52, no. 11 pp. 1980-1987, Nov. 2004

[10] J. Fuhl, A. Molish, and E. Bonek, "Unified channel model for mobile radio systems with smart antennas," Proc. Inst. Electr. Eng.-Radar, Sonar Navigation, vol. 145, no. 1, pp. 32-41, Feb. 1998.

[11] A. Paulraj, R. Nabar, and D. Gore, Introduction to Space-Time Wireless Communications. Cambridge, U.K.: Cambridge Univ. Press, 2003.

[12] Technical specification group radio access network; Spreading and modulation (FDD) (Release 6), 2003. 3rd Generation Partnership Project, 3GPP TS 25.213 V6.0.0.

[13] Technical specification group radio access network; Physical layer procedure (FDD) (Release 6), 2004. 3rd Generation Partnership Project, 3GPP TS 25.214 V6.2.0.

[14] S. Alamouti, "A simple transmit diversity technique for wireless communications," IEEE J. Sel. Areas Commun., vol. 16, no. 8, pp. 1451-1458, Oct. 1998.

[15] B. A. Bjerke, Z. Zvonar, and J. G. Proakis, "Antenna diversity combining schemes for WCDMA systems in fading multipath channels," IEEE Trans. Wireless Commun., vol. 3, no. 1, pp. 97-106, Jan. 2004.

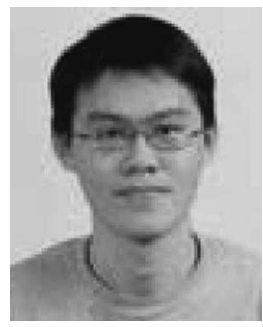

Po-Ying Chen (S'02) was born in Taipei, Taiwan, R.O.C., on January 20, 1979. He received the B.S. degree in electrical engineering and the $\mathrm{Ph} . \mathrm{D}$. degree in communication engineering from National Taiwan University, Taipei, in 2001 and 2006, respectively.

Since September 2006, he has been with Sunplus mMobile Inc., Hsinchu, Taiwan, R.O.C. His research interests include wireless channel characteristics, MIMO transmission techniques, and signal processing for high-speed wireless communications.

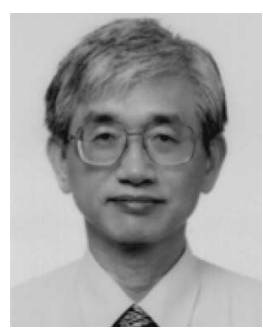

Hsueh-Jyh Li (S'84-M'87-SM'98) was born in Yun-Lin, Taiwan, R.O.C., on August 11, 1949. $\mathrm{He}$ received the B.S.E.E. degree from National Taiwan University, Taipei, Taiwan, in 1971 and the M.S.E.E. and the Ph.D. degrees from the University of Pennsylvania, Philadelphia, in 1980 and 1987, respectively.

Since 1973, he has been with the Department of Electrical Engineering, National Taiwan University, where he is a Professor. He was with the ElectronOptics and Microwave-Optics Laboratory, University of Pennsylvania, during 1984-1987. He was the Director of Communication Research Center during 1996-2000 and the Chairman of the Graduate Institute of Communication Engineering, National Taiwan University, during 1997-2000. His main research interests are in microstrip antennas, radar scattering, microwave imaging, radio channel characteristics, and wireless communication.

Prof. Li received the Distinguished Research Award from the National Science Council, R.O.C., in 1992 